\title{
From the reachable space of the heat equation to Hilbert spaces of holomorphic functions
}

\author{
Andreas Hartmann, Karim Kellay† Marius Tucsnak ${ }^{\ddagger}$
}

July 27, 2017

\begin{abstract}
This work considers systems described by the heat equation on the interval $[0, \pi]$ with $L^{2}$ boundary controls and it studies the reachable space at some instant $\tau>0$. The main results assert that this space is generally sandwiched between two Hilbert spaces of holomorphic functions defined on a square in the complex plane and which has $[0, \pi]$ as one of the diagonals. More precisely, in the case Dirichlet boundary controls acting at both ends we prove that the reachable space contains the Smirnov space and it is contained in the Bergman space associated to the above mentioned square. The methodology, quite different of the one employed in previous literature, is a direct one. We first represent the input-to-state map as an integral operator whose kernel is a sum of Gaussians and then we study the range of this operator by combining the theory of Riesz bases for Smirnov spaces in polygons and the theory developed by Aikawa, Hayashi and Saitoh on the range of integral transforms, in particular those associated with the heat kernel.
\end{abstract}

\section{Introduction}

We consider the system

$$
\left\{\begin{array}{lr}
\frac{\partial w}{\partial t}(t, x)=\frac{\partial^{2} w}{\partial x^{2}}(t, x) & t \geqslant 0, \\
w(t, 0)=u_{0}(t), \quad w(t, \pi)=u_{\pi}(t) & t \in[0, \pi), \\
w(0, x)=0 & x \in(0, \pi),
\end{array}\right.
$$

which models the heat propagation in a rod of length $\pi$, controlled by prescribing the temperature at both ends. It is well known (see, for instance, [9, Proposition 10.7.3]) that for every $u_{0}, u_{\pi} \in L^{2}[0, \infty)$ the problem (1.1) admits a unique solution $w \in C\left([0, \infty), W^{-1,2}(0, \pi)\right)$. (Recall that $W^{-1,2}(0, \pi)$ is the dual of the usual Sobolev space $W^{1,2}(0, \pi)$ with respect to

\footnotetext{
*Institut de Mathématiques de Bordeaux, Université de Bordeaux/Bordeaux INP/CNRS , 351 Cours de la Libération, 33405 TALENCE, France, andreas.hartman@u-bordeaux.fr

${ }^{\dagger}$ Institut de Mathématiques de Bordeaux, Université de Bordeaux/Bordeaux INP/CNRS, 351 Cours de la Libération, 33405 TALENCE, France, karim.kellay@u-bordeaux.fr

${ }^{\ddagger}$ Institut de Mathématiques de Bordeaux UMR 5251, Université de Bordeaux/Bordeaux INP/CNRS, 351 Cours de la Libération, 33405 TALENCE, France, marius.tucsnak@u-bordeaux.fr
} 
the pivot space $L^{2}[0, \pi]$.) Moreover, according to the same reference, the input-to-state maps $\left(\Phi_{\tau}\right)_{\tau>0}$ defined by

$$
\Phi_{\tau}\left[\begin{array}{l}
u_{0} \\
u_{\pi}
\end{array}\right]=w(\tau, \cdot) \quad\left(\tau>0, u_{0}, u_{\pi} \in L^{2}[0, \tau]\right)
$$

lie, for every $\tau>0$, in $\mathcal{L}\left(L^{2}([0, \tau] ; U), W^{-1,2}(0, \pi)\right)$. In control theoretic terms, this means that (1.1) defines a well-posed control system, with state space $X=W^{-1,2}(0, \pi)$ and input space $U=\mathbb{C}^{2}$. A classical important question in the study of control systems consists in the characterization of their reachable space at instant $\tau$, which is the space of states which can be attained at instant $\tau$ when the input is freely moving in $L^{2}([0, \tau] ; U)$. In our case this space is the range of $\Phi_{\tau}$, denoted $\operatorname{Ran} \Phi_{\tau}$, where $\Phi_{\tau}$ has been defined in (1.2). As far as we know, the first results on this space for the boundary controlled heat equation have been given in the classical paper of Fattorini and Russell [4], where it is shown that the functions which can be extended to holomorphic ones in a horizontal strip containing $[0, \pi]$ and which vanish, together with all their derivatives of even order, at $x=0$ and $x=\pi$, belong to $\operatorname{Ran} \Phi_{\tau}$. This result implies, in particular, that $\operatorname{Ran} \Phi_{\tau} \supset \operatorname{Ran} \mathbb{T}_{\tau}$, where $\mathbb{T}$ is the semigroup generated by the $1 D$ Dirichlet Laplacian in $W^{-1,2}(0, \pi)$, which means that the system determined by (1.1) is null-controllable in any time $\tau>0$. As remarked in Seidman [8] this means, in particular, that $\operatorname{Ran} \Phi_{\tau}$ is invariant with respect to $\tau>0$.

The first significant improvement of Fattorini's and Russell's result on this reachable space has been reported only in 2016, in the work by Martin, Rosier and Rouchon [7], where it has been shown that any function which can be extended to a function holomorphic in a certain disk in $\mathbb{C}$ containing the segment $[0, \pi]$ lies in the reachable space. This result has been further improved in Dardé and Ervedoza [2], where it has been shown that any function which can be extended to one which is holomorphic in a neighbourhood of the square $D$ defined by

$$
D=\{s=x+i y \in \mathbb{C} \quad|\quad| y \mid<x \text { and }|y|<\pi-x\},
$$

lies in the reachable space.

On the other hand, it is not difficult to check (see, for instance, [7, Theorem 1]) that if $\psi \in \operatorname{Ran} \Phi_{\tau}$ then $\psi$ can be extended to a function holomorphic in $D$, so that the assertion in [2] looks almost sharp. The aim of our work is to show that this result can be significantly improved, by showing that $\operatorname{Ran} \Phi_{\tau}$ can be sandwiched between two Hilbert spaces of analytic functions defined on $D$. The methodology we employ, completely different of those used in [7] or [2], is based on explicit series form representations of the solutions of (1.1), combined with results on Bergman and Smirnov spaces on $D$. For the sake of completeness, we recall the definitions of the above mentioned spaces. First, given an open set $\Omega \subset \mathbb{C}$, the Bergman space $A^{2}(\Omega)$ consists of all functions holomorphic in $\Omega$ with $\int_{\Omega}|f(x+i y)|^{2} \mathrm{~d} x \mathrm{~d} y<\infty$. Endowed with the norm induced from $L^{2}(\Omega), A^{2}(\Omega)$ is clearly a Hilbert space. We will also use weighted Bergman spaces. Let $\omega$ be a positive measurable function on $\Omega$, then $A^{2}(\Omega, \omega)$ is the space of holomorphic functions for which $\int_{\Omega}|f(x+i y)|^{2} \omega(x+i y) \mathrm{d} x \mathrm{~d} y<\infty$. The Smirnov space on a simply connected domain $\Omega$ can be defined provided that there is a conformal map $\varphi$ from the unit disk $\mathbb{D}$ to $\Omega$. If $\Gamma_{r}$ is the image under $\varphi$ of the circle $|z|=r$, then $f \in E^{2}(\Omega)$ if and only if $\sup _{r<1} \int_{\Gamma_{r}}|f(z)|^{2}|\mathrm{~d} z|<\infty$. The curves $\Gamma_{r}$ can be replaced by any sequence of rectifiable Jordan curves surrounding eventually every compact subdomain of $\Omega$ (see Duren [3]). If $\Omega$ is a Smirnov domain (in particular a polygon like $D$, see [5, Ch.VII,Thm. 4.6]) 
then, according to [3, p. 184]) we have that $f \in E^{2}(\Omega)$ iff $f \in L^{2}(\partial \Omega)$ with

$$
\int_{\partial \Omega} \zeta^{n} f(\zeta) \mathrm{d} \zeta=0 \quad(n \in \mathbb{N})
$$

In this case, the norm in $E^{2}(\Omega)$ is given by

$$
\|f\|_{E^{2}(\Omega)}^{2}=\int_{\partial \Omega}|f(\zeta)|^{2}|\mathrm{~d} \zeta|
$$

With the above definitions, the result in [7], asserting that $\operatorname{Ran} \Phi_{\tau} \subset \operatorname{Hol}(D)$ (the space formed by the functions holomorphic on $D$ ), can be strengthened to

Proposition 1.1. For every $\tau>0$ we have $\operatorname{Ran} \Phi_{\tau} \subset A^{2}(D)$.

Our main result improves the existing lower bounds of the reachable space and states as follows:

Theorem 1.2. For every $\tau>0$ we have $\operatorname{Ran} \Phi_{\tau} \supset E^{2}(D)$.

Remark 1.3. It turns out that the inclusion in Theorem 1.2 is strict (see Proposition 4.3 below). We do not have a similar result for the inclusion in Proposition 1.1. In view of the methodology used below, we conjecture that we have $\operatorname{Ran} \Phi_{\tau}=A^{2}(D)$.

The paper is organized as follows. In the next section we prove Proposition 1.1 using a series representation, based on the 1D heat equation kernel, of the input-to-state map. As it turns out below, the domain of holomorphy and the behavior of the sum of this series near the boundary of the holomorphy domain are determined by a dominating term, which is treated using a result by Aikawa, Hayashi and Saitoh on Laplace-type transforms having their range in Bergman spaces. In Section 3, following work by Levin-Lyubarskii, we introduce a Riesz basis of exponentials in the Smirnov space $E^{2}(D)$ which allows us to decompose functions in $E^{2}(D)$ into a sum of two functions in Bergman spaces associated with two infinite sectors. In Section 4 this allows us, in particular, to decompose functions in the Smirnov space $E^{2}(D)$ into a dominating term for which we use again Aikawa, Hayashi and Saitoh, and remainder terms which are "small" in appropriate weighted Bergman spaces on infinite sectors. Using a matrix type argument, this allows us to prove Theorem 1.2. In the last section we discuss the adaptations of our methods and results to some other boundary conditions and controls.

\section{Proof of Proposition 1.1}

Using the decomposition of the solution $w$ of (1.1) in the standard Fourier basis $(\sin (n x))_{n \geqslant 1}$ of $L^{2}[0, \pi]$, it is not difficult to check that the input to state maps $\left(\Phi_{\tau}\right)_{\tau \geqslant 0}$ defined in (1.2) write

$$
\begin{aligned}
\left(\Phi_{\tau} u\right)(x)=\sum_{n \geqslant 1} n & {\left[\int_{0}^{\tau} \mathrm{e}^{n^{2}(\sigma-\tau)} u_{0}(\sigma) \mathrm{d} \sigma\right] \sin (n x) } \\
& +\sum_{n \geqslant 1} n(-1)^{n+1}\left[\int_{0}^{\tau} \mathrm{e}^{n^{2}(\sigma-\tau)} u_{\pi}(\sigma) \mathrm{d} \sigma\right] \sin (n x) \quad(\tau>0, x \in(0, \pi)),
\end{aligned}
$$


where the above series converges in $W^{-1,2}(0, \pi)$, uniformly with respect to $\sigma \in[0, \tau]$. From the last formula it follows that

$$
\left(\Phi_{\tau} u\right)(x)=\int_{0}^{\tau} \frac{\partial K_{0}}{\partial x}(\tau-\sigma, x) u_{0}(\sigma) \mathrm{d} \sigma+\int_{0}^{\tau} \frac{\partial K_{\pi}}{\partial x}(\tau-\sigma, x) u_{\pi}(\sigma) \mathrm{d} \sigma \quad(x \in[0, \pi]),
$$

where

$$
\begin{aligned}
& K_{0}(\sigma, x)=-\sum_{n \geqslant 1} \mathrm{e}^{-n^{2} \sigma} \cos (n x) \\
&=-\frac{1}{2} \sum_{n \geqslant 1} \mathrm{e}^{-n^{2} \sigma}\left(\mathrm{e}^{i n x}+\mathrm{e}^{-i n x}\right)=-\frac{1}{2} \sum_{n \in \mathbb{Z}} \mathrm{e}^{-n^{2} \sigma} \mathrm{e}^{i n x} \quad(\sigma>0, \quad x \in(0, \pi)) . \\
& K_{\pi}(\sigma, x)=-K_{0}(\sigma, \pi-x) \quad(\sigma>0, \quad x \in(0, \pi)) .
\end{aligned}
$$

We note that for $\sigma>0$ we can extend $K_{0}(\sigma, \cdot)$ and $K_{\pi}(\sigma, \cdot)$ to functions in $L_{\text {loc }}^{2}(\mathbb{R})$ of period $2 \pi$. Formulas (2.5) and (2.6) have been widely used, often combined with duality arguments, to study the controllability properties of the system (1.1). However, it seems that this way of writing the kernels $K_{0}$ and $K_{\pi}$ is not very useful to prove reachability results beyond those classically obtained in [7]. We thus use an alternative form of $K_{0}$ and $K_{\pi}$, in terms of the heat kernel on the real line. For the sake of completeness, we derive below these formulae using the Poisson summation formula. Alternatively, these expressions of $K_{0}$ and $K_{\pi}$ could be obtained using the classical method of images, which allows deriving fundamental solutions of linear partial differential equations on a segment with appropriate boundary conditions from the corresponding fundamental solution for the same PDE on the whole line.

Proposition 2.1. We have

$$
\begin{aligned}
& K_{0}(\sigma, x)=-\frac{1}{2} \sqrt{\frac{\pi}{\sigma}} \sum_{m \in \mathbb{Z}} \mathrm{e}^{-\frac{(x+2 m \pi)^{2}}{4 \sigma}} \quad(\sigma>0, x \in \mathbb{R}), \\
& K_{\pi}(\sigma, x)=\frac{1}{2} \sqrt{\frac{\pi}{\sigma}} \sum_{m \in \mathbb{Z}} \mathrm{e}^{-\frac{(x+(2 m-1) \pi)^{2}}{4 \sigma}} \quad(\sigma>0, x \in \mathbb{R}),
\end{aligned}
$$

where the above series converge in $L^{2}[0, \pi]$.

Proof. We first remind the formula of the Fourier transform of a Gaussian, given by

$$
\widehat{G}_{\alpha}=\sqrt{\frac{\pi}{\alpha}} G_{\frac{1}{4 \alpha}} \quad(\alpha>0)
$$

where

$$
G_{\alpha}(y)=\mathrm{e}^{-\alpha y^{2}} \quad(y \in \mathbb{R}) .
$$

For $a>0, \gamma_{0}=\frac{2 \pi}{a}$ and $f \in \mathcal{S}(\mathbb{R})$, we also remind the Poisson summation formula in the form

$$
\sum_{m \in \mathbb{Z}} f(x+m a)=\frac{1}{a} \sum_{k \in \mathbb{Z}} \hat{f}\left(k \gamma_{0}\right) \mathrm{e}^{i k \gamma_{0} x} \quad(x \in \mathbb{R}) .
$$

We first apply (2.10) with $f(y)=\mathrm{e}^{-\frac{y^{2}}{4 \sigma}}$ and $a=2 \pi$, so that $\gamma_{0}=1$. Since, according to (2.9), we have

$$
\hat{f}(\xi)=\sqrt{2 \pi \sigma} \mathrm{e}^{-\sigma \xi^{2}} \quad(\xi \in \mathbb{R}),
$$


we obtain

$$
\frac{\sqrt{\pi}}{\sqrt{\sigma}} \sum_{m \in \mathbb{Z}} \mathrm{e}^{-\frac{(x+2 m \pi)^{2}}{4 \sigma}}=\sum_{n \in \mathbb{Z}} \mathrm{e}^{-\sigma n^{2}} \mathrm{e}^{i n x} \quad(x \in \mathbb{R}) .
$$

The above formula and (2.5) imply (2.7).

Finally, (2.8) follows from (2.7) and the fact that $K_{\pi}(\sigma, x)=-K_{0}(\sigma, \pi-x)$.

Our strategy to study $\operatorname{Ran} \Phi_{\tau}$ is to identify in each of the formulae for $K_{0}$ and $K_{\pi}$ in $(2.7)$ and (2.8) a principal term which corresponds, roughly speaking, to a boundary controlled heat equation on a half-line, and to show that the remaining terms can be seen as perturbations. To determine the range of the operators corresponding to the principal parts we use in an essential manner the following result from Aikawa, Hayashi and Saitoh [1].

Theorem 2.2. Let

$$
\Delta=\left\{s \in \mathbb{C} \mid-\frac{\pi}{4}<\arg s<\frac{\pi}{4}\right\} .
$$

For $s \in \Delta, \tau>0$ and $f \in L^{2}[0, \tau]$ we set

$$
\left(P_{\tau} f\right)(s)=\int_{0}^{\tau} \frac{s \mathrm{e}^{-\frac{s^{2}}{4(\tau-\sigma)}}}{2 \sqrt{\pi}(\tau-\sigma)^{\frac{3}{2}}} f(\sigma) \sqrt{\sigma} \mathrm{d} \sigma .
$$

Then $P_{\tau}$ defines an isometric isomorphism from $L^{2}[0, \tau]$ onto $A^{2}\left(\Delta, \omega_{0}\right)$, where

$$
\omega_{0}(s)=\frac{\mathrm{e}^{\frac{\mathrm{Res}^{2}}{2 \tau}}}{\tau} \quad(s \in \Delta) .
$$

A simple change of variables gives the following consequence of the above theorem.

Corollary 2.3. With the notation in Theorem 2.2, let

$$
\tilde{\Delta}=\pi-\Delta \text {. }
$$

For $s \in \tilde{\Delta}, \tau>0$ and $f \in L^{2}[0, \tau]$ we set

$$
\left(Q_{\tau} f\right)(s)=\int_{0}^{\tau} \frac{(\pi-s) \mathrm{e}^{-\frac{(\pi-s)^{2}}{4(\tau-\sigma)}}}{2 \sqrt{\pi}(\tau-\sigma)^{\frac{3}{2}}} f(\sigma) \sqrt{\sigma} \mathrm{d} \sigma .
$$

Then $Q_{\tau}$ defines an isometric isomorphism from $L^{2}[0, \tau]$ onto $A^{2}\left(\tilde{\Delta}, \omega_{\pi}\right)$, where

$$
\omega_{\pi}(s)=\frac{\mathrm{e}^{\frac{\operatorname{Re}(\pi-s)^{2}}{2 \tau}}}{\tau} \quad(s \in \pi-\Delta) .
$$

Remark 2.4. The operators $P_{\tau}$ ans $Q_{\tau}$ defined above are closely related to the derivative with respect to $x$ of the terms corresponding to $m=0$ in the definition (2.7) of $K_{0}$ and in the definition (2.8) of $K_{\pi}$, respectively. Alternatively, these operators can be connected to the heat equation on a half-line. More precisely, setting

$$
w_{l}(t, x)=\left(P_{t} f\right)(x) \quad(t \geqslant 0, x \geqslant 0),
$$


we have that

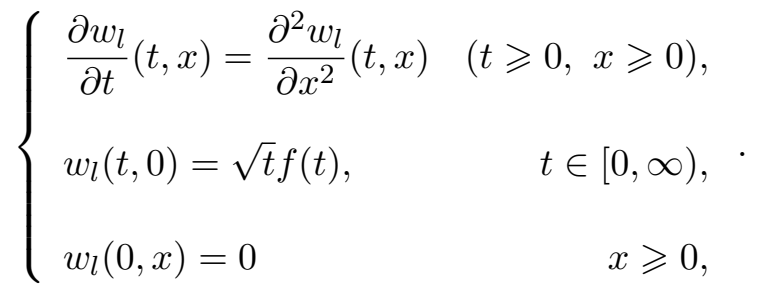

Similarly, setting

$$
w_{r}(t, x)=\left(Q_{t} f\right)(x) \quad(t \geqslant 0, x \leqslant \pi),
$$

we have that

$$
\left\{\begin{array}{lr}
\frac{\partial w_{r}}{\partial t}(t, x)=\frac{\partial^{2} w_{r}}{\partial x^{2}}(t, x) & (t \geqslant 0, x \leqslant \pi), \\
w_{r}(t, \pi)=\sqrt{t} f(t), & t \in[0, \infty), \\
w_{r}(0, x)=0 & x \geqslant 0,
\end{array} .\right.
$$

The presence of the factor $\sqrt{t}$ in the boundary conditions above (and consequently in the definition of $P_{\tau}$ and $Q_{\tau}$ ) is important in order to use the results in [1], where this factor allows using the explicit form of the reproducing kernel of $A^{2}(\Delta)$.

We next give a simple lemma to be used in the proof of Theorem 1.1.

Lemma 2.5. Let $\tau>0$ and $u \in L^{2}[0, \tau]$ and denote

$$
\varphi_{\tau}(s)=\int_{0}^{\tau} \frac{\mathrm{e}^{-\frac{s^{2}}{4(\tau-\sigma)}}}{(\tau-\sigma)^{3 / 2}} s u(\sigma) \mathrm{d} \sigma \quad(s \in D) .
$$

Then $\varphi_{\tau} \in A^{2}(D)$ and there exists a positive constant $C_{\tau}$ such that

$$
\left\|\varphi_{\tau}\right\|_{A^{2}(D)} \leqslant C_{\tau}\|u\|_{L^{2}[0, \tau]} \quad\left(u \in L^{2}[0, \tau]\right) .
$$

Proof. We first write $\varphi_{\tau}=\varphi_{\tau, 1}+\varphi_{\tau, 2}$, where

$$
\begin{array}{ll}
\varphi_{\tau, 1}(s)=\int_{0}^{\frac{\tau}{2}} \frac{\mathrm{e}^{-\frac{s^{2}}{4(\tau-\sigma)}}}{(\tau-\sigma)^{3 / 2}} s u(\sigma) \mathrm{d} \sigma & (s \in D), \\
\varphi_{\tau, 2}(s)=\int_{\frac{\tau}{2}}^{\tau} \frac{\mathrm{e}^{-\frac{s^{2}}{4(\tau-\sigma)}}}{(\tau-\sigma)^{3 / 2}} s u(\sigma) \mathrm{d} \sigma & (s \in D) .
\end{array}
$$

It is easy to check that

$$
\left\|\varphi_{\tau, 1}\right\|_{A^{2}(D)} \leqslant \tilde{C}_{\tau}\|u\|_{L^{2}[0, \tau]} \quad\left(u \in L^{2}[0, \tau]\right),
$$

for some positive constant $\tilde{C}_{\tau}$. We thus only have to estimate the norm of $\varphi_{\tau, 2}$ in $A^{2}(D)$. To this aim we define the following function on $[0, \tau]$ by

$$
\tilde{u}(t):=\left\{\begin{array}{lll}
0 & \text { if } \quad t \in[0, \tau / 2], \\
\frac{u(t)}{\sqrt{t}} & \text { if } \quad t \in[\tau / 2, \tau] .
\end{array}\right.
$$


Using Theorem 2.2, we get

$$
\begin{aligned}
\left\|\varphi_{\tau, 2}\right\|_{A^{2}(D)}^{2} & =4 \pi\left\|P_{\tau} \tilde{u}\right\|_{A^{2}(D)}^{2} \\
& \leqslant 4 \pi \tau\left\|P_{\tau} \tilde{u}\right\|_{A^{2}\left(\Delta, \omega_{0}\right)}^{2} \\
& =4 \pi \tau\|\tilde{u}\|_{L^{2}[0, \tau]}^{2} \\
& =4 \pi \tau \int_{\tau / 2}^{\tau} \frac{|u(\sigma)|^{2}}{\sigma} \mathrm{d} \sigma \\
& \leqslant 8 \pi\|u\|_{L^{2}[0, \tau]}^{2} .
\end{aligned}
$$

We are now in a position to give the main proof in this section.

Proof of Proposition 1.1. Let

$$
\tilde{K}_{0}(\sigma, s)=-\frac{1}{2} \sqrt{\frac{\pi}{\sigma}} \sum_{m \in \mathbb{Z}^{*}} \mathrm{e}^{-\frac{(s+2 m \pi)^{2}}{4 \sigma}} \quad(\sigma>0, s \in D),
$$

and

$$
\tilde{K}_{\pi}(\sigma, s)=\frac{1}{2} \sqrt{\frac{\pi}{\sigma}} \sum_{m \in \mathbb{Z}^{*}} \mathrm{e}^{-\frac{(s+(2 m-1) \pi)^{2}}{4 \sigma}} \quad(\sigma>0, s \in D) .
$$

Let

$$
\tilde{\psi}(x)=\int_{0}^{\tau} \frac{\partial \tilde{K}_{0}}{\partial x}(\tau-\sigma, x) u_{0}(\sigma) \mathrm{d} \sigma+\int_{0}^{\tau} \frac{\partial \tilde{K}_{\pi}}{\partial x}(\sigma, x) u_{\pi}(\sigma) \mathrm{d} \sigma \quad(x \in[0, \pi]) .
$$

By Lemma 2.5 it suffices to prove that $\tilde{\psi}$ can be extended to a function holomorphic in $A^{2}(D)$. To this aim, we note that there exists $a, b>0$ such that for every $k \in \mathbb{Z} \backslash\{-1,0\}$ we have

$$
\left|(s+k \pi) \mathrm{e}^{-\frac{(s+k \pi)^{2}}{4(\tau-\sigma)}}\right|^{2} \leqslant a k^{2} \mathrm{e}^{\frac{-b k^{2}}{(\tau-\sigma)}} \quad(s \in D) .
$$

It follows that for every $s \in D$ and for every $k \in \mathbb{Z} \backslash\{-1,0\}$ we have

$$
\int_{0}^{\tau} \frac{\left|(s+k \pi) \mathrm{e}^{-\frac{(s+k \pi)^{2}}{4(\tau-\sigma)}}\right|^{2}}{(\tau-\sigma)^{3}} \mathrm{~d} \sigma \leqslant a \int_{0}^{\tau} \frac{k^{2} \mathrm{e}^{\frac{-b k^{2}}{t}}}{t^{3}} \mathrm{~d} t=\frac{a}{b^{2} k^{2}} \int_{\frac{b k^{2}}{\tau}}^{\infty} u \mathrm{e}^{-u} \mathrm{~d} u \leqslant C\left(\frac{1}{\tau}+1\right) \mathrm{e}^{-\frac{b k^{2}}{\tau}} .
$$

The fact that $\tilde{\psi}$ is in $A^{2}(D)$ follows by combining the last estimate with the use of the Cauchy-Schwarz inequality in (2.17). This achieves the proof.

\section{$3 \quad$ A decomposition result in $E^{2}(D)$}

The aim of this section is to show that functions in $E^{2}(D)$ can be decomposed into a sum of two functions each of which being in a weighted Bergman space defined on an unbounded sector. An important ingredient in deriving this decomposition is a Riesz basis of exponentials in the Smirnov class $E^{2}(D)$, which is constructed using results from B. J. Levin and J. I. Ljubarskiu 


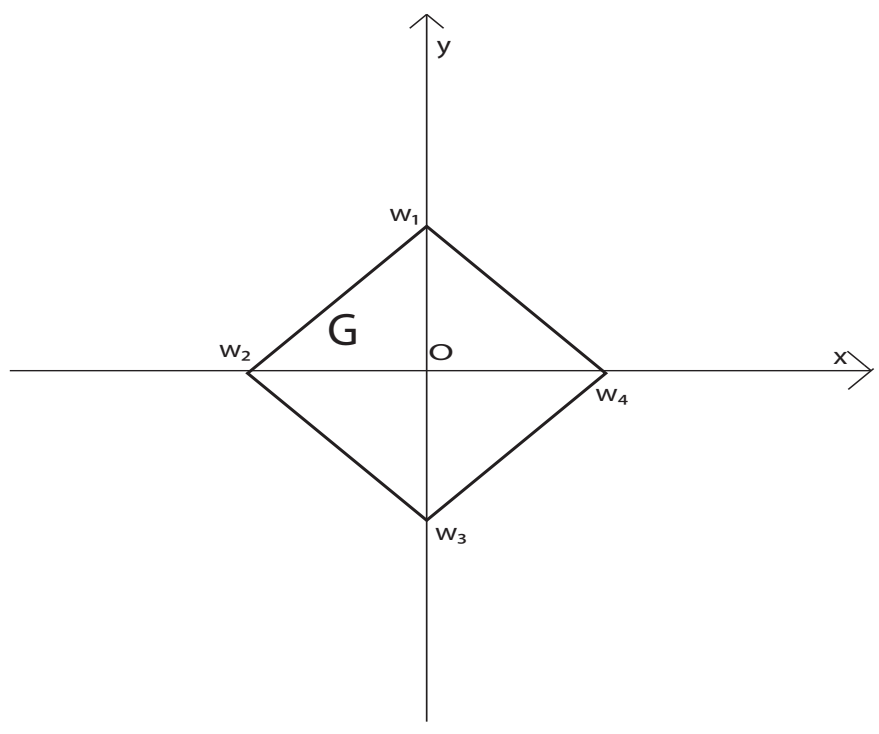

Figure 1: Square

[6]. To apply some of these results it is more convenient to replace $D$ by a square containing the origin. Denote

$$
\tilde{D}=-\frac{\pi}{2}+D
$$

To apply results from [6] we need some notation. Let

$$
w_{1}=\frac{\pi}{2} i, w_{2}=-\frac{\pi}{2}, w_{3}=-\frac{\pi}{2} i, w_{4}=\frac{\pi}{2},
$$

be the vertices of the square $\tilde{D}$ (see Figure 1 ).

For $j \in\{2,3,4\}$ we denote by $l_{j}$ the edge joining $w_{j-1}$ and $w_{j}$, whereas $l_{1}$ is the edge joining $w_{4}$ to $w_{1}$. We also denote by $\theta_{j}$ the angle formed by the exterior normal to $l_{j}$ with the positive sense of the $O x$ axis, so that

$$
\theta_{1}=\frac{\pi}{4}, \theta_{2}=\frac{3 \pi}{4}, \theta_{3}=\frac{5 \pi}{4}, \theta_{4}=\frac{7 \pi}{4} .
$$

We also define (see Figure 2) the sectors

$$
\Gamma_{j}=\left\{\lambda \in \mathbb{C} \mid \arg \lambda \in\left(\theta_{j}, \theta_{j+1}\right)\right\} .
$$

Consider the angular support function of $\tilde{D}$ defined by $H(\lambda)=\operatorname{Re}\left(\overline{w_{j}} \lambda\right)$ for $\lambda \in \Gamma_{j}$. We thus have

$$
H(\lambda)=\frac{1}{2} \begin{cases}\pi \operatorname{Im} \lambda & \text { for } \lambda \in \Gamma_{1}, \\ -\pi \operatorname{Re} \lambda & \text { for } \lambda \in \Gamma_{2}, \\ -\pi \operatorname{Im} \lambda & \text { for } \lambda \in \Gamma_{3}, \\ \pi \operatorname{Re} \lambda & \text { for } \lambda \in \Gamma_{4} .\end{cases}
$$

For $j \in\{1,2,3,4\}$ and $K>0$ we set

$$
\Pi_{j}(K)=\left\{\lambda \in \mathbb{C}\left|\operatorname{Re}\left(\lambda \mathrm{e}^{-i \theta_{j}}\right)>0, \quad\right| \operatorname{Im}\left(\lambda \mathrm{e}^{-i \theta_{j}}\right) \mid<K\right\} .
$$




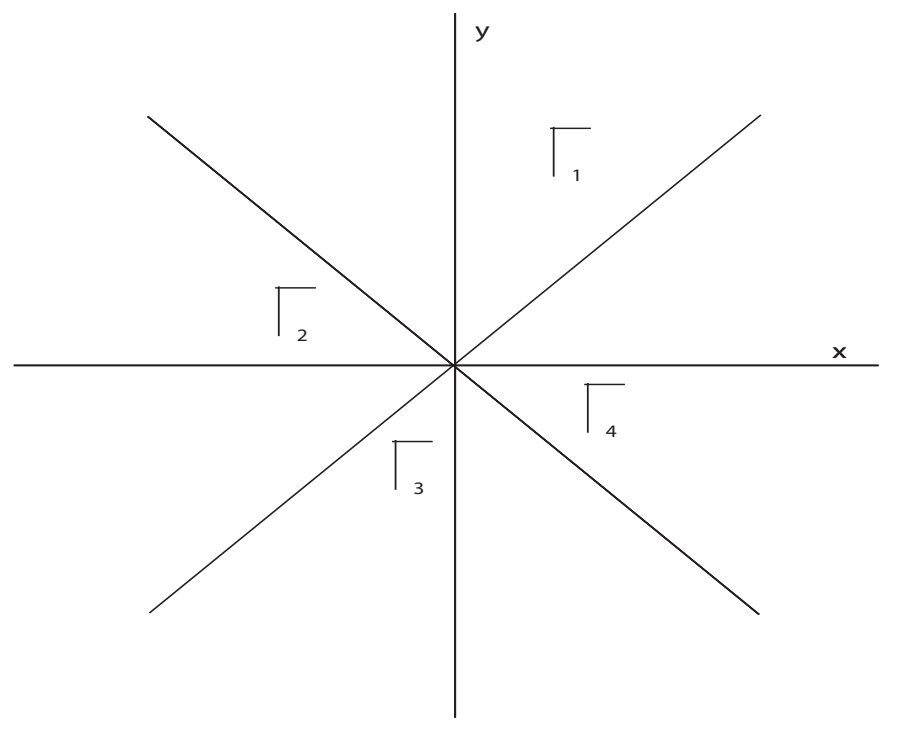

Figure 2: Gamma

Finally, for every $K>0$ we introduce

$$
\tilde{D}_{K}=\cup_{j=1}^{4} \Pi_{j}(K) .
$$

The above notation has been used in [6] to introduce a class of functions playing an important role in the construction of Riesz bases of exponentials in Smirnov spaces defined on general convex polygons containing the origin. For the sake of simplicity, we recall below some definitions and results from [6] when the polygon is chosen to be the above defined square $\tilde{D}$.

Definition 3.1. The class of functions $S_{\tilde{D}}$ is formed by the functions $S$ which are holomorphic on $\tilde{D}$, of exponential type and

1. The function $S$ has a sequence $\left(\lambda_{k}\right)_{k \in \mathbb{Z}}$ of simple zeros and there exists $K>0$ such that all these zeros lie in $\tilde{D}_{K}$;

2. $\inf _{j \neq k}\left|\lambda_{j}-\lambda_{k}\right|=2 \delta>0$;

3. There exist $c, C>0$ such that

$$
0<c<|S(\lambda)| \mathrm{e}^{-H(\lambda)}<C \quad\left(\lambda \notin \tilde{D}_{K}\right) .
$$

Theorem 3.2. With the notation in Definition 3.1, the family $\left(\mathrm{e}^{\lambda_{k} z} \mathrm{e}^{-H\left(\lambda_{k}\right)}\right)_{k \in \mathbb{Z}}$ is a Riesz basis in $E^{2}(\tilde{D})$.

With the change of variable behind (3.1) in mind, we obtain the following consequence of Theorem 3.2:

Corollary 3.3. With the above notation, the family $\left(g_{\lambda}\right)_{\lambda \in \Lambda}$ defined by

$$
g_{\lambda}(s)=\mathrm{e}^{\lambda s} \mathrm{e}^{-\frac{\lambda \pi}{2}} \mathrm{e}^{-H(\lambda)} \quad(\lambda \in \Lambda, s \in \mathbb{C}),
$$

is a Riesz basis in $E^{2}(D)$. 
With the indicator function of $\tilde{D}$ given by (3.4), as asserted in [6], a function $S$ satisfying the conditions in Definition 3.1 is

$$
S(\lambda)=\sum_{j=1}^{4} \mathrm{e}^{\overline{w_{j}} \lambda} \quad(\lambda \in \mathbb{C}),
$$

where $w_{j}$ are defined in (3.2). More precisely, we have

$$
\begin{aligned}
S(\lambda) & =\mathrm{e}^{\frac{\pi}{2} \lambda}+\mathrm{e}^{-\frac{\pi}{2} \lambda}+\mathrm{e}^{i \frac{\pi}{2} \lambda}+\mathrm{e}^{-i \frac{\pi}{2} \lambda} \\
& =2 \cos \left(\frac{\pi}{2} \lambda\right)+2 \cos \left(i \frac{\pi}{2} \lambda\right) \\
& =4 \cos \left(\frac{\pi(1+i) \lambda}{4}\right) \cos \left(\frac{\pi(1-i) \lambda}{4}\right) \quad(\lambda \in \mathbb{C}) .
\end{aligned}
$$

The zeros of $S$ form the set $\Lambda$ defined by

$$
\Lambda=\{(2 k+1)(1 \pm i): k \in \mathbb{Z}\}
$$

We next use the Riesz basis constructed above to show that functions in $E^{2}(D)$ can be written as sum of two functions, each of which is in a Bergman space defined on an infinite sector. To this aim, let

$$
\Delta=\left\{s \in \mathbb{C} \mid-\frac{\pi}{4}<\arg s<\frac{\pi}{4}\right\} .
$$

Lemma 3.4. Let $\Lambda$ be the sequence introduced in (3.7) and let the polynomial function $p$ be defined by

$$
p(s)=s+2 \pi i \quad(s \in \mathbb{C}) .
$$

Let $\varphi \in E^{2}(D)$ with $\varphi=\sum_{\lambda \in \Lambda} a_{\lambda} g_{\lambda}$. Denote

$$
\varphi_{1}=\sum_{\substack{\lambda \in \Lambda \\ \operatorname{Re} \lambda<0}} a_{\lambda} g_{\lambda} \quad \text { and } \quad \varphi_{2}=\sum_{\substack{\lambda \in \Lambda \\ \operatorname{Re} \lambda>0}} a_{\lambda} g_{\lambda} .
$$

Then $\varphi_{1} / p \in A^{2}(\Delta)$ et $\varphi_{2} / p \in A^{2}(\pi-\Delta)$.

Proof. We first note that using (3.4) it follows that

$$
\left|\mathrm{e}^{-\frac{(2 k+1)(1 \pm i) \pi}{2}} \mathrm{e}^{-H((2 k+1)(1 \pm i))}\right|=1 \quad(k \leqslant-1) .
$$

Moreover, for every $k \leqslant-1$ and $x+i y \in \Delta$ we have

$$
\left|\mathrm{e}^{(2 k+1)(1+i)(x+i y)}\right|=\mathrm{e}^{(2 k+1)(x-y)}, \quad\left|\mathrm{e}^{(2 k+1)(1-i)(x+i y)}\right|=\mathrm{e}^{(2 k+1)(x+y)},
$$

so that

$$
\left|\mathrm{e}^{\lambda(x+i y)}\right| \leqslant \mathrm{e}^{(\operatorname{Re} \lambda)(x-|y|)} \quad(x+i y \in \Delta, \lambda \in \Lambda, \operatorname{Re} \lambda<0) .
$$

We also note that there exists $c>0$ such that

$$
|p(x+i y)|^{2} \geqslant \frac{1}{2}\left(x^{2}+c\right) \quad(x+i y \in \Delta) .
$$

Indeed $|p(x+i y)|^{2}-\frac{1}{2} x^{2}=\frac{1}{2} x^{2}+(y+2 \pi)^{2}$ is vanishing only in the point $(0,-2 \pi)$, which is far away from $\Delta \cup(\pi-\Delta)$, so that on this set it is bounded from below by a strictly positive 
constant. Combining (3.12) and (3.11) it follows that for every $\lambda, \mu \in \Lambda$ with $\operatorname{Re} \lambda<0$ and $\operatorname{Re} \mu<0$ we have:

$$
\begin{aligned}
\left|\left\langle\frac{g_{\lambda}}{p}, \frac{g_{\mu}}{p}\right\rangle_{A^{2}(\Delta)}\right| & \leqslant \int_{\Delta} \frac{\left|\mathrm{e}^{\lambda s}\right|\left|\mathrm{e}^{\mu s}\right|}{|p(s)|^{2}} \mathrm{~d} x \mathrm{~d} y \\
& \leqslant \int_{\Delta} \frac{\mathrm{e}^{(x-|y|)(\operatorname{Re} \lambda+\operatorname{Re} \mu)}}{|p(x+i y)|^{2}} \mathrm{~d} x \mathrm{~d} y \\
& \leqslant 4 \int_{0}^{+\infty} \frac{\mathrm{e}^{x(\operatorname{Re} \lambda+\operatorname{Re} \mu)}}{x^{2}+c}\left(\int_{0}^{x} \mathrm{e}^{y(-\operatorname{Re} \lambda-\operatorname{Re} \mu)} \mathrm{d} y\right) \mathrm{d} x \\
& =\frac{4}{-\operatorname{Re} \lambda-\operatorname{Re} \mu} \int_{0}^{+\infty} \frac{\mathrm{e}^{x(\operatorname{Re} \lambda+\operatorname{Re} \mu)}\left(\mathrm{e}^{x(-\operatorname{Re} \lambda-\operatorname{Re} \mu)}-1\right)}{x^{2}+c} \mathrm{~d} x \\
& \leqslant \frac{4}{-\operatorname{Re} \lambda-\operatorname{Re} \mu} \int_{0}^{+\infty} \frac{\mathrm{d} x}{x^{2}+c} \\
& \leqslant C \frac{1}{(|\operatorname{Re} \lambda|+|\operatorname{Re} \mu|)},
\end{aligned}
$$

for a suitable constant $C$. Using Hilbert's inequality it follows that

$$
\begin{aligned}
\left\|\frac{\varphi_{1}}{p}\right\|_{A^{2}(\Delta)}^{2} & =\sum_{\substack{\lambda, \mu \in \Lambda \\
\operatorname{Re} \lambda<0 \operatorname{Re} \mu<0}} a_{\lambda} \overline{a_{\mu}}\left\langle\frac{g_{\lambda}}{p}, \frac{g_{\mu}}{p}\right\rangle_{A^{2}(\Delta)} \\
& \leqslant \frac{C}{2} \sum_{\substack{\lambda, \mu \in \Lambda \\
\operatorname{Re} \lambda<0 \operatorname{Re} \mu<0}} \frac{\left|a_{\lambda}\right|\left|a_{\mu}\right|}{|\operatorname{Re} \lambda|+|\operatorname{Re} \mu|} \\
& \leqslant \frac{C \pi}{2} \sum_{\substack{\lambda \in \Lambda \\
\operatorname{Re} \lambda<0}}\left|a_{\lambda}\right|^{2},
\end{aligned}
$$

which shows that $\varphi_{1} / p \in A^{2}(\Delta)$.

To prove that $\varphi_{2} / p \in A^{2}(\pi-\Delta)$, let $\lambda \in \Lambda$, with $\operatorname{Re} \lambda>0$ and remark that

$$
g_{\lambda}(\pi-s)=g_{-\lambda}(s) \quad(s \in \Delta) .
$$

Denoting $q(s):=p(\pi-s)$, we have

$$
|q(x+i y)|^{2}=|p(\pi-s)|^{2}=(\pi-x)^{2}+(y-2 \pi)^{2} \geqslant \frac{1}{10}\left(x^{2}+d\right) \quad(x+i y \in \Delta),
$$

for a suitably chosen $d>0$. Indeed, arguing similarly as above we have

$$
|\pi-s+2 \pi|^{2}-\frac{1}{10} x^{2}=\frac{9}{10}\left(x-\frac{10}{9} \pi\right)^{2}+(y-2 \pi)^{2}-\frac{1}{9} \pi^{2} \quad(x+i y \in \Delta) .
$$

The right-hand side of the above equality vanishes on an ellipse which stays strictly away from $\Delta$ and hence it admits a strictly positive minimum there. Consequently, for every $\lambda, \mu \in \Lambda$, 
with $\operatorname{Re} \lambda, \operatorname{Re} \mu>0$ we have

$$
\begin{aligned}
\left|\left\langle\frac{g_{\lambda}}{p}, \frac{g_{\mu}}{p}\right\rangle_{A^{2}(\pi-\Delta)}\right| & =\left|\left\langle\frac{g_{-\lambda}}{q}, \frac{g_{-\mu}}{q}\right\rangle_{A^{2}(\Delta)}\right| \\
& \leqslant \int_{\Delta} \frac{\mathrm{e}^{(x-|y|)(-\operatorname{Re} \lambda-\operatorname{Re} \mu)}}{|q(x+i y)|^{2}} \mathrm{~d} x \mathrm{~d} y \\
& \leqslant 20 \int_{0}^{+\infty} \frac{\mathrm{e}^{x(-\operatorname{Re} \lambda-\operatorname{Re} \mu)}}{x^{2}+d}\left(\int_{0}^{x} \mathrm{e}^{y(\operatorname{Re} \lambda+\operatorname{Re} \mu)} \mathrm{d} y\right) \mathrm{d} x \\
& \leqslant \frac{C}{\operatorname{Re} \lambda+\operatorname{Re} \mu},
\end{aligned}
$$

where $C$ is a constant possibly different from the preceding case. Using, as above, Hilbert's inequality, we conclude that $\varphi_{2} / p \in A^{2}(\pi-\Delta)$, which ends the proof.

At this point we recall, given an open set $\Omega \subset \mathbb{C}$, the classical notation $H^{\infty}(\Omega)$, which stands for the space of bounded analytic functions in $\Omega$.

Lemma 3.5. Let $\eta>0$ and let $p$ be defined by (3.9). Then there exists $f \in \operatorname{Hol}(\Delta \cup(\pi-\Delta))$ satisfying the following conditions

(i) $\frac{p(s) \mathrm{e}^{\eta s^{2}}}{f(s)} \in H^{\infty}(\Delta)$,

(ii) $\frac{p(s) \mathrm{e}^{\eta(\pi-s)^{2}}}{f(s)} \in H^{\infty}(\pi-\Delta)$.

Proof. We first note that if suffices to construct a function $g$ such that

(i') $\frac{1}{g(s)} \in H^{\infty}(\Delta)$

(ii') $\frac{\mathrm{e}^{-2 \pi \eta s}}{g(s)} \in H^{\infty}(\pi-\Delta)$

Indeed, assuming the existence of $g$ with the above properties, we see that $f(s)=\mathrm{e}^{\eta s^{2}} g(s) p(s)$ satisfies the requirements in the conclusion of the lemma.

We show below that the function $g$ defined by

$$
g(s)=\frac{\cosh (c s)}{r(s)} \quad(s \in \Delta \cup(\pi-\Delta)),
$$

where $c>2 \pi \eta$ and $r$ is an appropriately chosen polynomial, satisfies the above conditions. More precisely, let $r$ be defined by

$$
r(s)=\prod_{\mu \in \mathcal{I}}(s-\mu) \quad(s \in \mathbb{C})
$$

where

$$
\left.\mathcal{I}=\left\{-\frac{i}{c}\left(n+\frac{1}{2}\right) \pi\right)\right\}_{n \in \mathbb{Z}} \cap[-i \pi, i \pi] .
$$


Note that $\mathcal{I}$ is the set formed by the roots of $\cosh (c s)$ on the segment $[-i \pi, i \pi]$. Clearly, $g \in \operatorname{Hol}(\Delta \cup(\pi-\Delta))$ and $1 / g \in \operatorname{Hol}(\Delta \cup(\pi-\Delta))$.

We still have to show that $g$ satisfies conditions (i') and (ii') above. To this aim, it clearly suffices to show that

$$
|g(s)| \geqslant \text { Const } \mathrm{e}^{2 \pi \eta|x|}, \quad s=x+i y \in \Delta \cup(\pi-\Delta) .
$$

To check the above estimate, let $s=x+i y \in \Delta \cup(\pi-\Delta)$, with $|x| \geqslant \frac{1}{c}$. Then

$$
|\cosh (c s)|^{2}=\frac{1}{2^{2}}\left|\mathrm{e}^{c(x+i y)}+\mathrm{e}^{-c(x+i y)}\right|^{2}=\frac{1}{4}\left(\mathrm{e}^{2 c x}+\mathrm{e}^{-2 c x}+2 \cos (2 y)\right) \geqslant \frac{1}{4}\left(\mathrm{e}^{2 c|x|}-2\right),
$$

It follows that there exists $C>0$ such that

$$
|\cosh (c s)| \geqslant C \mathrm{e}^{c|x|} \quad\left(s=x+i y \in \Delta \cup(\pi-\Delta),|x| \geqslant \frac{1}{c}\right) .
$$

Denoting by $N$ the degree of $r$ we have

$$
|r(s)| \leqslant \text { Const }|s|^{N} \leqslant \text { Const }(1+|x|)^{N}, \quad s \in \Delta \cup(\pi-\Delta) .
$$

Hence, recalling that $c>2 \pi \eta$, we obtain, for $s=x+i y \in \Delta \cup(\pi-\Delta)$ with $|x| \geqslant \frac{1}{c}$, that

$$
|g(s)|=\frac{|\cosh (c s)|}{|r(s)|} \geqslant \text { Const } \frac{\mathrm{e}^{c|x|}}{1+|x|^{N}} \geqslant \text { Const } \mathrm{e}^{2 \pi \eta|x|} .
$$

For $s=x+i y \in \Delta \cup(\pi-\Delta)$ with $|x| \leqslant \frac{1}{c}, g$ is non-vanishing, so that, using compactness, it is bounded from below by a strictly positive constant.

We have thus proved (3.13) which, as explained above, implies the conclusion of the lemma.

Corollary 3.6. With the notation in Lemma 3.4, let $\tau>0$ and $\varphi \in E^{2}(D)$. Then there exists $\varphi_{1} \in A^{2}\left(\Delta, \omega_{0}\right), \varphi_{2} \in A^{2}\left(\pi-\Delta, \omega_{\pi}\right)$, where the weights $\omega_{0}$ and $\omega_{\pi}$ are those defined in (2.12) and (2.14), respectively, such that

$$
\varphi(s)=\varphi_{1}(s)+\varphi_{2}(s) \quad(s \in D) .
$$

Proof. Let $\eta=\frac{1}{4 \tau}$ and let $f$ be the corresponding function constructed in Lemma 3.5. This implies that the mappings $s \mapsto \frac{p(s) \mathrm{e}^{s^{2}}}{f(s)}$ and $s \mapsto \frac{p(s) \mathrm{e}^{(\pi-s)^{2}}}{f(s)}$, with $p$ defined in (3.9), are in $H^{\infty}(\Delta)$ and $H^{\infty}(\pi-\Delta)$, respectively. The function $s \mapsto f(s) \varphi(s)$ clearly lies in $E^{2}(D)$. According to Lemma 3.4 we have $f \varphi=\varphi_{1,0}+\varphi_{2,0}$, with $\frac{\varphi_{1,0}}{p} \in A^{2}(\Delta)$ and $\frac{\varphi_{2,0}}{p} \in A^{2}(\pi-\Delta)$. Setting $\psi_{k}=\varphi_{k, 0} / p$, we thus get

$$
\frac{f(s) \varphi(s)}{p(s)}=\psi_{1}(s)+\psi_{2}(s) \quad(s \in D),
$$

with $\psi_{1} \in A^{2}(\Delta), \psi_{2} \in A^{2}(\pi-\Delta)$. Define $\varphi_{1}, \varphi_{2}$ by

$$
\varphi_{1}(s)=\frac{p(s)}{f(s)} \psi_{1}(s) \quad(s \in \Delta)
$$




$$
\varphi_{2}(s)=\frac{p(s)}{f(s)} \psi_{2}(s) \quad(s \in \pi-\Delta) .
$$

We clearly have $\varphi=\varphi_{1}+\varphi_{2}$ in $D$. Moreover, using Lemma 3.5 (recall that we have chosen $\eta=1 / 4 \tau)$, we have that the map

$$
s \mapsto \varphi_{1}(s) \mathrm{e}^{s^{2} / 4 \tau}=\frac{p(s) \mathrm{e}^{s^{2} /(4 \tau)}}{f(s)} \psi_{1}(s)
$$

lies in $A^{2}(\Delta)$. Similarly, the map

$$
s \mapsto \varphi_{2}(s) \mathrm{e}^{(\pi-s)^{2} / 4 \tau}=\frac{P(s) \mathrm{e}^{(\pi-s)^{2} /(4 \tau)}}{f(s)} \psi_{1}(s)
$$

lies in $A^{2}(\pi-\Delta)$, which concludes the proof.

\section{Proof of the main result}

Let $\tilde{K}_{0}$ and $\tilde{K}_{\pi}$ be the functions introduced in (2.15) and (2.16), respectively. We decompose these functions as

$$
\tilde{K}_{0}(\sigma, s)=A(\sigma, s)+B(\sigma, s), \quad \text { and } \quad \tilde{K}_{\pi}(\sigma, s)=C(\sigma, s)+D(\sigma, s),
$$

where

$$
\begin{aligned}
& A(\sigma, s)=-\frac{1}{2} \sqrt{\frac{\pi}{\sigma}} \sum_{m \geqslant 1} \mathrm{e}^{-\frac{(s+2 m \pi)^{2}}{4 \sigma}} \quad \text { and } \quad C(\sigma, s)=\frac{1}{2} \sqrt{\frac{\pi}{\sigma}} \sum_{m \geqslant 1} \mathrm{e}^{-\frac{(s+(2 m-1) \pi)^{2}}{4 \sigma}} \\
& B(\sigma, s)=-\frac{1}{2} \sqrt{\frac{\pi}{\sigma}} \sum_{m \leqslant-1} \mathrm{e}^{-\frac{(s+2 m \pi)^{2}}{4 \sigma}} \quad \text { and } \quad D(\sigma, s)=\frac{1}{2} \sqrt{\frac{\pi}{\sigma}} \sum_{m \leqslant-1} \mathrm{e}^{-\frac{(s+(2 m-1) \pi)^{2}}{4 \sigma}} \text {. }
\end{aligned}
$$

Denote

$$
R_{A, \tau} u(s)=\int_{0}^{\tau} \frac{\partial A}{\partial s}(\tau-\sigma, s) \sqrt{\sigma} u(\sigma) d \sigma,
$$

and similarly $R_{B, \tau}, R_{C, \tau}$ and $R_{D, \tau}$.

Lemma 4.1. Let $\omega_{0}$ and $\omega_{\pi}$ be the weights defined in (2.12) and (2.14), respectively. There exists an absolute constant $C_{1}>0$ such that for every $u \in L^{2}[0, \tau]$ we have

1. $R_{A, \tau} u \in A^{2}\left(\Delta, \omega_{0}\right)$ and

$$
\left\|R_{A, \tau} u\right\|_{A^{2}\left(\Delta, \omega_{0}\right)} \leqslant C_{1} \sqrt{\tau(1+\tau)^{3}} \frac{\mathrm{e}^{-\pi^{2} / \tau}}{1-\mathrm{e}^{-\pi^{2} / \tau}}\|u\|_{L^{2}[0, \tau]} \quad(\tau>0) .
$$

2. $R_{B, \tau} u \in A^{2}\left(\pi-\Delta, \omega_{\pi}\right)$ and

$$
\left\|R_{B, \tau} u\right\|_{A^{2}\left(\pi-\Delta, \omega_{\pi}\right)} \leqslant C_{1} \sqrt{\tau(1+\tau)^{3}} \frac{\mathrm{e}^{-\pi^{2} / 4 \tau}}{1-\mathrm{e}^{-\pi^{2} / 2 \tau}}\|u\|_{L^{2}[0, \tau]} \quad(\tau>0) .
$$

3. $R_{C, \tau} u \in A^{2}\left(\Delta, \omega_{0}\right)$ and

$$
\left\|R_{C, \tau} u\right\|_{A^{2}\left(\Delta, \omega_{0}\right)} \leqslant C_{1} \sqrt{\tau(1+\tau)^{3}} \frac{\mathrm{e}^{-\pi^{2} / 4 \tau}}{1-\mathrm{e}^{-\pi^{2} / 2 \tau}}\|u\|_{L^{2}[0, \tau]} \quad(\tau>0) .
$$


4. $R_{D, \tau} u \in A^{2}\left(\pi-\Delta, \omega_{\pi}\right)$ and

$$
\left\|R_{D, \tau} u\right\|_{A^{2}\left(\pi-\Delta, \omega_{\pi}\right)} \leqslant C_{1} \sqrt{\tau(1+\tau)^{3}} \frac{\mathrm{e}^{-\pi^{2} / \tau}}{1-\mathrm{e}^{-\pi^{2} / \tau}}\|u\|_{L^{2}[0, \tau]} \quad(\tau>0) .
$$

Proof. Let

$$
\rho_{m}(s, \tau)= \begin{cases}\int_{0}^{\tau} \frac{\sqrt{\pi}}{4(\tau-\sigma)^{3 / 2}}(s+m \pi) \mathrm{e}^{-\frac{(s+m \pi)^{2}}{4(\tau-\sigma)}} \sqrt{\sigma} u(\sigma) \mathrm{d} \sigma \quad m \geqslant 1, s \in \Delta, \\ \int_{0}^{\tau} \frac{\sqrt{\pi}}{4(\tau-\sigma)^{3 / 2}}(s+m \pi) \mathrm{e}^{-\frac{(s+m \pi)^{2}}{4(\tau-\sigma)}} \sqrt{\sigma} u(\sigma) \mathrm{d} \sigma \quad m \leqslant-1, s \in \pi-\Delta .\end{cases}
$$

By the triangular and the Cauchy-Schwarz inequality we see that for $m \geqslant 1$ we have

$$
\begin{aligned}
& \left\|\rho_{m}(\cdot, \tau)\right\|_{A^{2}\left(\Delta, \omega_{0}\right)}^{2} \\
& \quad=\frac{1}{\tau} \iint_{\Delta} \mathrm{e}^{\frac{x^{2}-y^{2}}{2 \tau}}\left|\int_{0}^{\tau} \frac{\sqrt{\pi}}{4(\tau-\sigma)^{3 / 2}}(s+m \pi) \mathrm{e}^{-\frac{(s+m \pi)^{2}}{4(\tau-\sigma)}} \sqrt{\sigma} u(\sigma) \mathrm{d} \sigma\right|^{2} \mathrm{~d} x \mathrm{~d} y \\
& \quad \leqslant \frac{\pi\|u\|_{L^{2}(0, \tau)}^{2}}{16} \iint_{\Delta}\left(|x+m \pi|^{2}+y^{2}\right) \mathrm{e}^{\frac{x^{2}-y^{2}}{2 \tau}} \int_{0}^{\tau} \frac{\mathrm{e}^{-\frac{(x+m \pi)^{2}-y^{2}}{2(\tau-\sigma)}}}{(\tau-\sigma)^{3}} \mathrm{~d} \sigma \mathrm{d} x \mathrm{~d} y \\
& \quad=\frac{\pi\|u\|_{L^{2}(0, \tau)}^{2}}{16} \iint_{\Delta}\left(|x+m \pi|^{2}+y^{2}\right) \mathrm{e}^{\frac{x^{2}-y^{2}}{2 \tau}} \int_{0}^{\tau} \frac{\mathrm{e}^{-\frac{(x+m \pi)^{2}-y^{2}}{2 \sigma}}}{\sigma^{3}} \mathrm{~d} \sigma \mathrm{d} x \mathrm{~d} y .
\end{aligned}
$$

Set $\alpha=\frac{(x+m \pi)^{2}-y^{2}}{2}$. Recalling that $m \geqslant 1$ we see that

$$
\alpha=\frac{(x+m \pi)^{2}-y^{2}}{2}=\frac{x^{2}-y^{2}+2 m \pi x+m^{2} \pi^{2}}{2} \geqslant \frac{\pi^{2}}{2} m^{2} \geqslant 1 .
$$

This, together with the change of variables $u=\alpha / \sigma$ and an integration by parts, yields

$$
\begin{aligned}
\int_{0}^{\tau} \frac{\mathrm{e}^{-\frac{(x+m \pi)^{2}-y^{2}}{2 \sigma}}}{\sigma^{3}} \mathrm{~d} \sigma \mathrm{d} x \mathrm{~d} y & =\frac{1}{\alpha^{2}} \int_{\alpha / \tau}^{\infty} u \mathrm{e}^{-u} \mathrm{~d} u=\frac{\mathrm{e}^{-\alpha / \tau}}{\alpha^{2}}(1+\alpha / \tau) \\
& \leqslant \frac{\mathrm{e}^{-\alpha / \tau}}{\alpha}(1+1 / \tau) .
\end{aligned}
$$

Using (4.1), the above estimate implies that

$$
\begin{aligned}
& \left\|\rho_{m}(\cdot, \tau)\right\|_{A^{2}\left(\Delta, \omega_{0}\right)}^{2}=\frac{1}{\tau} \iint_{\Delta} \mathrm{e}^{\frac{x^{2}-y^{2}}{2 \tau}}\left|\rho_{m}(x+i y, \tau)\right|^{2} \mathrm{~d} x \mathrm{~d} y \\
& \quad \leqslant \frac{\pi}{16}\|u\|_{L^{2}[0, \tau]}^{2}\left(1+\frac{1}{\tau}\right) \iint_{\Delta} \mathrm{e}^{\frac{x^{2}-y^{2}}{2 \tau}} \frac{(x+m \pi)^{2}+y^{2}}{(x+m \pi)^{2}-y^{2}} \mathrm{e}^{-\frac{x^{2}+2 m \pi x+m^{2} \pi^{2}-y^{2}}{2 \tau}} \mathrm{d} x \mathrm{~d} y .
\end{aligned}
$$

Since $|y| \leqslant x$ for $s=x+i y \in \Delta$ we have

$$
\frac{(x+m \pi)^{2}+y^{2}}{(x+m \pi)^{2}-y^{2}}=1+\frac{2 y^{2}}{(x+m \pi)^{2}-y^{2}} \leqslant 1+\frac{2 x^{2}}{m^{2} \pi^{2}} \leqslant 1+x^{2} \quad(m \geqslant 1, x+i y \in \Delta) .
$$


The last two estimates imply that for every $m \geqslant 1$ we have

$$
\begin{aligned}
\left\|\rho_{m}(\cdot, \tau)\right\|_{A^{2}\left(\Delta, \omega_{0}\right)}^{2} & \leqslant \frac{\pi}{16}\|u\|_{L^{2}[0, \tau]}^{2}\left(1+\frac{1}{\tau}\right) \iint_{\Delta} \mathrm{e}^{\frac{x^{2}-y^{2}}{2 \tau}}\left(1+x^{2}\right) \mathrm{e}^{-\frac{x^{2}+2 m \pi x+m^{2} \pi^{2}-y^{2}}{2 \tau}} \mathrm{d} x \mathrm{~d} y \\
& =\frac{\pi}{16}\|u\|_{L^{2}[0, \tau]}^{2}\left(1+\frac{1}{\tau}\right) \mathrm{e}^{-m^{2} \pi^{2} / 2 \tau} \int_{x>0} \int_{|y| \leqslant x}\left(1+x^{2}\right) \mathrm{e}^{-m \pi x / \tau} \mathrm{d} y \mathrm{~d} x \\
& \leqslant \frac{\pi}{16}\|u\|_{L^{2}[0, \tau]}^{2}\left(1+\frac{1}{\tau}\right) \mathrm{e}^{-m^{2} \pi^{2} / 2 \tau} \int_{x>0} 2 x\left(1+x^{2}\right) \mathrm{e}^{-m \pi x / \tau} \mathrm{d} x .
\end{aligned}
$$

By the change of variables $u=x / \tau$ the last integral in the above formula can be estimated by

$$
\int_{x>0} 2 x\left(1+x^{2}\right) \mathrm{e}^{-m \pi x / \tau} \mathrm{d} x=\int_{u>0} 2 u \tau\left(1+u^{2} \tau^{2}\right) \mathrm{e}^{-m \pi u} \tau \mathrm{d} u \leqslant C \tau^{2}\left(1+\tau^{2}\right),
$$

where, in view of the crude estimate $m \geqslant 1$, the constant $C$ is independent on $\tau>0$ and on $m \geqslant 1$. Consequently,

$$
\left\|\rho_{m}(\cdot, \tau)\right\|_{A^{2}\left(\Delta, \omega_{0}\right)} \leqslant C_{1}\|u\|_{L^{2}[0, \tau]} \sqrt{\tau(1+\tau)^{3}} \mathrm{e}^{-m^{2} \frac{\pi^{2}}{4 \tau}} \quad(m \geqslant 1, \tau>0),
$$

where $C_{1}=\frac{\sqrt{C \pi}}{4}$. We are now in a position to prove estimates 1 and 3 . To this aim, remark that

$$
R_{A, \tau} u(s)=\sum_{m \geqslant 1} \rho_{2 m}(s, \tau), \quad R_{C, \tau} u(s)=\sum_{m \geqslant 1} \rho_{2 m-1}(s, \tau) \quad(\tau>0, s \in \Delta) .
$$

Using (4.2) and making the rough estimate $\mathrm{e}^{-m^{2} \pi^{2} / 4 \tau} \leqslant \mathrm{e}^{-m \pi^{2} / 4 \tau}$, we obtain

$$
\begin{aligned}
\left\|R_{A, \tau} u\right\|_{A^{2}\left(\Delta, \omega_{0}\right)} & \leqslant C_{1}\|u\|_{L^{2}[0, \tau]} \sqrt{\tau(1+\tau)^{3}} \sum_{m \geq 1} \mathrm{e}^{-(2 m)^{2} \pi^{2} / 4 \tau} \\
& \leqslant C_{1}\|u\|_{L^{2}[0, \tau]} \sqrt{\tau(1+\tau)^{3}} \sum_{m \geq 1} \mathrm{e}^{-m \pi^{2} / \tau} \\
& =C_{1}\|u\|_{L^{2}[0, \tau]} \sqrt{\tau(1+\tau)^{3}} \frac{\mathrm{e}^{-\pi^{2} / \tau}}{1-\mathrm{e}^{-\pi^{2} / \tau}},
\end{aligned}
$$

which implies 1. Similarly, for every $\tau>0$ we have

$$
\begin{aligned}
\left\|R_{C, \tau} u\right\|_{A^{2}\left(\Delta, \omega_{0}\right)} & \leqslant C_{1}\|u\|_{L^{2}[0, \tau]} \sqrt{\tau(1+\tau)^{3}} \sum_{m \geq 1} \mathrm{e}^{-(2 m-1)^{2} \pi^{2} / 4 \tau} \\
& \leqslant C_{1}\|u\|_{L^{2}[0, \tau]} \sqrt{\tau(1+\tau)^{3}} \mathrm{e}^{\pi^{2} / 4 \tau} \sum_{m \geqslant 1} \mathrm{e}^{-\frac{m \pi^{2}}{2 \tau}} \\
& =C_{1}\|u\|_{L^{2}[0, \tau]} \sqrt{\tau(1+\tau)^{3}} \frac{\mathrm{e}^{-\frac{\pi^{2}}{4 \tau}}}{1-\mathrm{e}^{-\pi^{2} / 2 \tau}} .
\end{aligned}
$$

To prove assertions 2 and 4 in the lemma, it suffices to remark that, using the change of 
variables $s=\pi-\tilde{s}, \tilde{s} \in \pi-\Delta$, for every $m \leqslant-2$ we have

$$
\begin{aligned}
& \left\|\rho_{m}(\cdot, \tau)\right\|_{A^{2}\left(\pi-\Delta, \omega_{\pi}\right)}^{2} \\
& \quad=\frac{1}{\tau} \iint_{\pi-\Delta}\left|\mathrm{e}^{(\pi-\tilde{s})^{2} / 4 \tau} \int_{0}^{\tau} \frac{\sqrt{\pi}}{4(\tau-\sigma)^{3 / 2}}(\tilde{s}+m \pi) \mathrm{e}^{-\frac{(\tilde{s}+m \pi)^{2}}{4(\tau-\sigma)}} \sqrt{\sigma} u(\sigma) \mathrm{d} \sigma\right|^{2} \mathrm{~d} x \mathrm{~d} y \\
& \quad=\frac{1}{\tau} \iint_{\Delta}\left|\mathrm{e}^{s^{2} / 4 \tau} \int_{0}^{\tau} \frac{\sqrt{\pi}}{4(\tau-\sigma)^{3 / 2}}(\pi-s+m \pi) \mathrm{e}^{-\frac{(\pi-s+m \pi)^{2}}{4(\tau-\sigma)}} \sqrt{\sigma} u(\sigma) \mathrm{d} \sigma\right|^{2} \mathrm{~d} x \mathrm{~d} y \\
& \quad=\frac{1}{\tau} \iint_{\Delta}\left|\mathrm{e}^{s^{2} / 4 \tau} \int_{0}^{\tau} \frac{\sqrt{\pi}}{4(\tau-\sigma)^{3 / 2}}(s+(|m|-1) \pi) \mathrm{e}^{-\frac{(s+(|m|-1) \pi)^{2}}{4(\tau-\sigma)}} \sqrt{\sigma} u(\sigma) \mathrm{d} \sigma\right|^{2} \mathrm{~d} x \mathrm{~d} y \\
& \quad=\left\|\rho_{|m|-1}(\cdot, \tau)\right\|_{A^{2}\left(\Delta, \omega_{0}\right)}^{2} \cdot
\end{aligned}
$$

Consequently the estimates on $R_{B, \tau}$ and $R_{D, \tau}$ follow exactly as those of $R_{C, \tau}$ and $R_{A, \tau}$, respectively.

We are now in a position to prove the main result of the paper.

Proof of Theorem 1.2. For every $\tau>0$ we introduce the operator

$$
M_{\tau}:=\left[\begin{array}{cc}
P_{\tau}+R_{A, \tau} & R_{C, \tau} \\
R_{B, \tau} & Q_{\tau}+R_{D, \tau}
\end{array}\right],
$$

where $P_{\tau}$ and $Q_{\tau}$ have been introduced in (2.11) and (2.13), respectively, whereas $R_{A, \tau}, R_{B, \tau}$, $R_{C, \tau}$ and $R_{D, \tau}$ have been defined at the beginning of this section. According to Lemma 4.1, we have

$$
M_{\tau} \in \mathcal{L}\left(\left(L^{2}([0, \pi])\right)^{2}, A^{2}\left(\Delta, \omega_{0}\right) \times A^{2}\left(\pi-\Delta, \omega_{\pi}\right)\right) \quad(\tau>0) .
$$

According to Theorem 2.2 and Corollary 2.3 we have that the operator

$$
\left[\begin{array}{cc}
P_{\tau} & 0 \\
0 & Q_{\tau}
\end{array}\right] \in \mathcal{L}\left(\left(L^{2}([0, \pi])\right)^{2}, A^{2}\left(\Delta, \omega_{0}\right) \times A^{2}\left(\pi-\Delta, \omega_{\pi}\right)\right) \quad(\tau>0),
$$

is invertible and

$$
\left\|\left[\begin{array}{cc}
P_{\tau} & 0 \\
0 & Q_{\tau}
\end{array}\right]\right\|_{\mathcal{L}\left(\left(L^{2}([0, \pi])\right)^{2}, A^{2}\left(\Delta, \omega_{0}\right) \times A^{2}\left(\pi-\Delta, \omega_{\pi}\right)\right)}=1 \quad(\tau>0) .
$$

On the other hand, using Lemma 4.1 we have that

$$
\lim _{\tau \rightarrow 0+}\left\|\left[\begin{array}{ll}
R_{A, \tau} & R_{C, \tau} \\
R_{B, \tau} & R_{D, \tau}
\end{array}\right]\right\|_{\mathcal{L}\left(\left(L^{2}([0, \pi])\right)^{2}, A^{2}\left(\Delta, \omega_{0}\right) \times A^{2}\left(\pi-\Delta, \omega_{\pi}\right)\right)}=0 .
$$

The last two norm estimates and (4.3) imply that there exists $\tau^{*}>0$ such that $M_{\tau}$ is invertible, at least for every $\tau \in\left(0,2 \tau^{*}\right)$.

Let now $\varphi \in E^{2}(D)$. By Corollary 3.6 (with $\tau=\tau^{*}$ ), there exists a decomposition

$$
\varphi(s)=\varphi_{1}(s)+\varphi_{2}(s) \quad(s \in D),
$$

where $\varphi_{1}$ and $\varphi_{2}$ are in the weighted Bergman spaces $A^{2}\left(\Delta, \omega_{0}^{*}\right)$ and $A\left(\pi-\Delta, \omega_{\pi}^{*}\right)$, where $\omega_{0}^{*}$ and $\omega_{\pi}^{*}$ are defined by (2.12) and (2.14) with $\tau=\tau^{*}$, respectively. Since $M_{\tau^{*}}$ is invertible there exist $v_{0}, v_{\pi}$ in $L^{2}\left(\left[0, \tau^{*}\right]\right)$ such that $M_{\tau^{*}}\left[\begin{array}{l}v_{0} \\ v_{\pi}\end{array}\right]=\left[\begin{array}{l}\varphi_{1} \\ \varphi_{2}\end{array}\right]$. Setting

$$
u_{0}(\sigma)=\sqrt{\sigma} v_{0}(\sigma), \quad u_{\pi}(\sigma)=\sqrt{\sigma} v_{\pi}(\sigma) \quad\left(\sigma \in\left[0, \tau^{*}\right]\right),
$$


and comparing the formulae (2.4), (2.7) and (2.8) for $\Phi_{\tau}$ with the definitions of the operators $P_{\tau}, Q_{\tau}, R_{A, \tau}, R_{B, \tau}, R_{C, \tau}$ and $R_{D, \tau}$ we see that for every $s \in D$

$$
\left(\Phi_{\tau^{*}}\left[\begin{array}{l}
u_{0} \\
u_{\tau}
\end{array}\right]\right)(s)=\underbrace{\left[\left(P_{\tau}+R_{A, \tau}\right)\left(v_{0}\right)\right](s)+\left[R_{C, \tau}\left(v_{\pi}\right)\right](s)}_{\varphi_{1}(s)}+\underbrace{\left[R_{B, \tau}\left(v_{0}\right)\right](s)+\left[\left(Q_{\tau}+R_{D, \tau}\right)\left(v_{\pi}\right)\right](s)}_{\varphi_{2}(s)},
$$

which, together with (4.4), shows that $\operatorname{Ran} \Phi_{\tau^{*}} \supset E^{2}(D)$. The conclusion follows using the fact, already mentioned in the Introduction, that $\operatorname{Ran} \Phi_{\tau}$ is independent of $\tau>0$.

Remark 4.2. Note that the sum of remainder terms defines a function which is holomorphic in a neighborhood of $D$, so that the direct perturbation argument above could be replaced by an application of the main result in [2].

We finish this section with a counter-example showing that there are functions in the range of $\Phi_{\tau}$ which are not in the Smirnov space $E^{2}(D)$. Looking to the proof of Theorem 1.2 , we readily see that it is sufficient to construct a function which decomposes into a sum of two functions in the corresponding weighted Bergman spaces and which is not in the Smirnov space.

Proposition 4.3. Let $1 / 2<\alpha<1$. Then

$$
\varphi(s)=\frac{e^{-\frac{s^{2}}{4 \tau}}}{s^{\alpha}(1+s)^{2}}+\frac{e^{-\frac{(\pi-s)^{2}}{4 \tau}}}{(2 \pi-s)^{2}} \in \operatorname{Ran} \Phi_{\tau} \backslash E^{2}(D) .
$$

Proof. Let us show that $\varphi \notin E^{2}(D)$. Since $1 /(2 \pi-s)^{2}, 1 /(1+s)^{2}, e^{-\frac{s^{2}}{4 \tau}}$ and $e^{-\frac{(\pi-s)^{2}}{4 \tau}}$ are holomorphic and non-vanishing on a neighborhood of $D$, it suffices to prove that $\psi(s)=$ $1 / s^{\alpha} \notin E^{2}(D)$. Since $\alpha>1 / 2$, we have

$$
\int_{\partial D} \frac{|d \zeta|}{|\zeta|^{2 \alpha}} \asymp \int_{0}^{1} \frac{d x}{x^{2 \alpha}}=\infty
$$

we conclude $\psi \notin L^{2}(\partial D)$ and therefore $\psi \notin E^{2}(D)$. As a result $\varphi \notin E^{2}(D)$.

Let us now show that $\varphi \in \operatorname{Ran} \Phi_{\tau}$. We have

$$
\varphi(s)=\frac{e^{-\frac{s^{2}}{4 \tau}}}{s^{\alpha}(1+s)^{2}}+\frac{e^{-\frac{(\pi-s)^{2}}{4 \tau}}}{(2 \pi-s)^{2}}=e^{-\frac{s^{2}}{4 \tau}} \varphi_{1}(s)+e^{-\frac{(\pi-s)^{2}}{4 \tau}} \varphi_{2}(s), \quad s \in D .
$$

By Corollary 3.6, It is enough to show that $\varphi_{1} \in A^{2}(\Delta)$ and $\varphi_{2} \in A^{2}(\pi-\Delta)$. Note that with the change of variables $s=x+i y=\pi-\tilde{s}, \tilde{s} \in \pi-\Delta$, we get

$$
\left\|\varphi_{2}\right\|_{A^{2}(\pi-\Delta)}^{2}=\int_{\Delta} \frac{\mathrm{d} x \mathrm{~d} y}{|\pi+x+i y|^{4}}=\int_{0}^{\infty} \int_{|y| \leqslant x} \frac{\mathrm{d} x \mathrm{~d} y}{\left((x+\pi)^{2}+y^{2}\right)^{2}} \leqslant \int_{0}^{\infty} \frac{2 x}{(x+\pi)^{4}} \mathrm{~d} x<\infty
$$

which shows $\varphi_{2} \in A^{2}(\pi-\Delta)$. Now, since $2 \alpha-1<1$, we get

$$
\begin{aligned}
\left\|\varphi_{1}\right\|_{A^{2}(\Delta)}^{2} & =\int_{\Delta} \frac{\mathrm{d} x \mathrm{~d} y}{|x+i y|^{2 \alpha}|1+x+i y|^{4}}=\int_{0}^{\infty} \int_{|y| \leqslant x} \frac{\mathrm{d} x \mathrm{~d} y}{\left(x^{2}+y^{2}\right)^{\alpha}\left((x+1)^{2}+y^{2}\right)^{2}} \\
& \leqslant \int_{0}^{\infty} \frac{2 x}{x^{2 \alpha}(x+1)^{4}} \mathrm{~d} x<\infty,
\end{aligned}
$$

and $\varphi_{1} \in A^{2}(\Delta)$. 


\section{Other boundary conditions}

In this section we discuss the adaptations of the results and methods above to the case of controls which act at only one end of the segment $[0, \pi]$ and/or are exerted via Neumann boundary condition instead of the Dirichlet ones.

We first consider a particular case of the system (1.1), obtained by taking $u_{\pi}=0$, i.e.,

$$
\left\{\begin{array}{lll}
\frac{\partial w}{\partial t}(t, x)=\frac{\partial^{2} w}{\partial x^{2}}(t, x) & t \geqslant 0, & x \in(0, \pi), \\
w(t, 0)=u_{0}(t), w(t, \pi)=0 & t \in[0, \infty), \\
w(0, x)=0 & x \in(0, \pi) .
\end{array}\right.
$$

As previously, given $\tau>0$, we are interested in the range of the input to state map $\Phi_{\tau}^{0}$ defined by

$$
\Phi_{\tau}^{0} u_{0}=w(\tau, \cdot) \quad\left(u_{0} \in L^{2}[0, \tau]\right) .
$$

With the notation and results in Section 2, we clearly have that

$$
\begin{aligned}
\left(\Phi_{\tau}^{0} u_{0}\right)(x)=\left(\Phi_{\tau}\right. & {\left.\left[\begin{array}{c}
u_{0} \\
0
\end{array}\right]\right)(x) } \\
& =\int_{0}^{\tau} \frac{\partial K_{0}}{\partial x}(\tau-\sigma, x) u_{0}(\sigma) \mathrm{d} \sigma \quad\left(\tau>0, x \in[0, \pi], u_{0} \in L^{2}[0, \tau]\right) .
\end{aligned}
$$

where $\Phi_{\tau}$ and $K_{0}$ have been defined in (1.2) and (2.7), respectively. Using the $2 \pi$ periodicity of $K_{0}$ and a parity argument, it is easily seen that, at least if $u_{0}$ is smooth and $u_{0}(0)=0$, the function $x \mapsto\left(\Phi_{\tau}^{0} u_{0}\right)(x)$ can be extended to a smooth function $\psi_{\tau}$, defined on $[0,2 \pi]$ by

$$
\begin{aligned}
\psi_{\tau}(x)=\frac{1}{2} \int_{0}^{\tau}\left(\frac{\partial K_{0}}{\partial x}(\tau-\sigma, x)-\frac{\partial K_{0}}{\partial x}(\tau-\sigma, 2 \pi-x)\right) & u_{0}(\sigma) \mathrm{d} \sigma \\
& \left(\tau>0, x \in[0,2 \pi], u_{0} \in L^{2}[0, \tau]\right) .
\end{aligned}
$$

The above formula, combined with slight variations of Proposition 1.1 and of Theorem 1.2 yields:

Proposition 5.1. Let $\tau>0$ and let $\Phi_{\tau}^{0}$ be the operator defined in (5.6). Denote

$$
\begin{aligned}
G & =\{s=x+i y \in \mathbb{C}|| y \mid<x \text { and }|y|<2 \pi-x\}, \\
\tilde{A}^{2}(G) & =\left\{\psi \in A^{2}(G) \mid \psi(s)+\psi(2 \pi-s)=0 \text { for all } s \in G\right\}, \\
\tilde{E}^{2}(G) & =\left\{\psi \in E^{2}(G) \mid \psi(s)+\psi(2 \pi-s)=0 \text { for all } s \in G\right\} .
\end{aligned}
$$

Then

$$
\tilde{E}^{2}(G) \subset \operatorname{Ran} \Phi_{\tau}^{0} \subset \tilde{A}^{2}(G) .
$$

The reachable space for the $1 D$ heat equation with Neumann boundary controls at both ends can be easily obtained from the reachable space for the Dirichlet boundary control at 
both ends. Indeed, consider the system, with state trajectory $\theta$ and control functions $u_{0}, u_{\pi}$, described by the equations

$$
\left\{\begin{array}{lr}
\frac{\partial \theta}{\partial t}(t, x)=\frac{\partial^{2} \theta}{\partial x^{2}}(t, x) & t \geqslant 0, \\
\frac{\partial \theta}{\partial x}(t, 0)=u_{0}(t), \quad \frac{\partial \theta}{\partial x}(t, \pi)=u_{\pi}(t) & t \in[0, \infty), \\
\theta(0, x)=0 & x \in(0, \pi) .
\end{array}\right.
$$

It is easy to check that, given $\tau>0$ and $u_{0}, u_{\pi} \in L^{2}[0, \tau]$, a function $\theta \in C\left([0, \tau] ; L^{2}[0, \pi]\right)$ satisfies (5.8) iff $w:=\frac{\partial \theta}{\partial x}$ satisfies (1.1). Combining this fact with Proposition 1.1 and Theorem 1.2 we obtain :

Proposition 5.2. Let $D$ be the set defined in (1.3), let $\tau>0$ and let $\Phi_{\tau}^{N N} \in \mathcal{L}\left(L^{2}[0, \tau] ; L^{2}[0, \pi]\right)$ be the input-yo-state map of (5.8), defined by

$$
\Phi_{\tau}^{N N}\left[\begin{array}{l}
u_{0} \\
u_{\pi}
\end{array}\right]=\theta(\tau, \cdot) \quad\left(u_{0}, u_{\pi} \in L^{2}[0, \tau]\right)
$$

Then

$$
E^{2,1}(D) \subset \operatorname{Ran} \Phi_{\tau}^{N N} \subset W^{2,1}(D) \cap \operatorname{Hol}(D),
$$

where $E^{2,1}(D)$ is the Smirnov-Sobolev space defined by

$$
E^{2,1}(D)=\left\{f \in \operatorname{Hol}(D) \mid f^{\prime} \in E^{2}(D)\right\},
$$

and $W^{2,1}(D)$ is the usual Sobolev space.

The space $W^{2,1}(D) \cap \operatorname{Hol}(D)$ is also known as the Dirichlet space.

Finally, we consider the case in which the Dirichlet boundary control at $x=0$ is replaced by a Neumann one, whereas the boundary condition at $x=\pi$ is a Dirichlet homogeneous one. More precisely, we consider the system

$$
\left\{\begin{array}{lrl}
\frac{\partial w}{\partial t}(t, x)=\frac{\partial^{2} w}{\partial x^{2}}(t, x) & t \geqslant 0, & x \in(0, \pi), \\
\frac{\partial w}{\partial x}(t, 0)=u_{0}(t), w(t, \pi)=0 & & t \in[0, \infty), \\
w(0, x)=0 & x \in(0, \pi) .
\end{array}\right.
$$

This time we are interested in the range of the input to state map $\Phi_{\tau}^{N}$ defined by

$$
\Phi_{\tau}^{N} u_{0}=w(\tau, \cdot) \quad\left(u_{0} \in L^{2}[0, \tau]\right) .
$$

After calculations which can be performed similarly to the Dirichlet boundary control case we are led to determine the range of the operator

$$
\left(\Phi_{\tau}^{N} u_{0}\right)(x)=\int_{0}^{\tau} K^{N}(\tau-\sigma, x) u_{0}(\sigma) \mathrm{d} \sigma \quad(\tau \geqslant 0, x \in[0, \pi]),
$$


where $G$ has been defined in (5.7), and

$$
K^{N}(\sigma, s)=\frac{\sqrt{\pi}}{2 \sqrt{2 \sigma}}\left[\sum_{m \in \mathbb{Z}} \mathrm{e}^{-\frac{(s-4 m \pi)^{2}}{4 \sigma}}-\sum_{m \in \mathbb{Z}} \mathrm{e}^{-\frac{(s-(4 m-2) \pi)^{2}}{4 \sigma}}\right] \quad(\sigma>0, s \in G) .
$$

Note that, unlike in the case of Dirichlet boundary control, the heat kernel is not derivated with respect to $x$ in the definition of the operator we are aiming to discuss the range. Therefore, we expect this range to contain more regular functions. Indeed, using slight variations of the methods developed above it can be proved that:

Proposition 5.3. Let $\tau>0$ and let $\Phi_{\tau}^{N}$ be the operator defined in (5.11). Using the notation in Proposition 5.1, we introduce the spaces

$$
\begin{aligned}
& \tilde{\tilde{A}}^{2}(G)=\left\{\psi \in \operatorname{Hol}(G) \quad \mid \psi^{\prime} \in \tilde{A}^{2}(G)\right\}, \\
& \tilde{\tilde{E}}^{2}(G)=\left\{\psi \in \operatorname{Hol}(G) \quad \mid \psi^{\prime} \in \tilde{E}^{2}(G)\right\} .
\end{aligned}
$$

Then

$$
\tilde{\tilde{E}}^{2}(G) \subset \operatorname{Ran} \Phi_{\tau}^{N} \subset \tilde{\tilde{A}}^{2}(G)
$$

Acknowledgements. The authors are grateful to S. Ervedoza and S. Micu for helpful discussions and suggestions on the first versions of this work. The research of first and the second authors was supported by the Austrian-French AMADEUS project 35598VB and the joint French-Russian Research Project PRC CNRS/RFBR 2017-2019. The third author acknowledges the support of the IFSMACS grant financed by Agence Nationale de la Recherche, reference ANR-15-CE40-0010.

\section{References}

[1] H. Aikawa, N. Hayashi, And S. Saitoh, The Bergman space on a sector and the heat equation, Complex Variables Theory Appl., 15 (1990), pp. 27-36.

[2] J. DARdÉ And S. ERvedozA, On the reachable set for the one-dimensional heat equation. working paper or preprint, Sept. 2016.

[3] P. L. Duren, Theory of $H^{p}$ spaces, Pure and Applied Mathematics, Vol. 38, Academic Press, New York-London, 1970.

[4] H. O. Fattorini And D. L. Russell, Exact controllability theorems for linear parabolic equations in one space dimension, Arch. Rational Mech. Anal., 43 (1971), pp. 272-292.

[5] J. B. Garnett and D. E. Marshall, Harmonic measure, vol. 2 of New Mathematical Monographs, Cambridge University Press, Cambridge, 2005.

[6] B. J. LeVIn AND J. I. LJUBARSKII, Interpolation by entire functions belonging to special classes and related expansions in series of exponentials, Izv. Akad. Nauk SSSR Ser. Mat., 39 (1975), pp. 657-702, 704.

[7] P. Martin, L. Rosier, And P. Rouchon, On the reachable states for the boundary control of the heat equation, Appl. Math. Res. Express. AMRX, (2016), pp. 181-216. 
[8] T. I. Seidman, Time-invariance of the reachable set for linear control problems, J. Math. Anal. Appl., 72 (1979), pp. 17-20.

[9] M. TUCSNAK AND G. Weiss, Observation and control for operator semigroups, Birkhäuser Advanced Texts: Basler Lehrbücher. [Birkhäuser Advanced Texts: Basel Textbooks], Birkhäuser Verlag, Basel, 2009. 